\title{
Scientific Realism and Historical Evidence: Shortcomings of the Current State of Debate
}

\author{
Juha Saatsi \\ 29th March 2010 \\ Department of Philosophy, \\ University of Leeds \\ Leeds LS2 9JT, UK \\ J.T.Saatsi@leeds.ac.uk
}

\begin{abstract}
This paper discusses three shortcomings of the current state of the debate regarding historical evidence against scientific realism. Attending to these issues will direct the debate away from over-generalising wholesale arguments.
\end{abstract}

\section{Introduction}

The scientific realism debate is a venerable one. Recently, however, some philosophers have grown tired of the debate, given the terms in which it is typically construed. Magnus and Callender (2004), for example, express a feeling of ennui afflicting the continuing dispute around scientific realism. The debate, framed in terms of "wholesale" arguments that concern "all or most of mature science", is arguably irresolvable. I'm sympathetic to their rejection of wholesale arguments, but my grounds for such rejection are very different from those of Magnus and Callender. I don't see reason to dismiss the use of historical evidence in the scientific realism debate altogether, but there are some hitherto unappreciated reasons to reconsider the way in which historical evidence bears on the realism debate. This paper identifies three quite general shortcomings of the current state of the realism debate that demand further attention and will direct the debate away from overgeneralising wholesale arguments. These shortcomings are: 
1. SCAntiness Of CASE-STUdies: historical evidence has not been sought extensively, open-mindedly, and across the board, partly because it is not even clear exactly what kinds of historical case-studies matter.

2. Disparity of explanatory Considerations: Realist strategies for dealing with historical evidence are often out of sync with their basic motivation (the Miracles argument).

3. Over generalisation: Positions in the realism debate have been construed too rigidly and in overly general terms, ignoring the potential for domain-specific (or 'relativized') (anti-)realist theses.

I proceed by explicating these shortcomings in this order.

\section{Scantiness of case-studies}

Most realist commentaries on historical theory-shifts focus on just one or two oft-repeated cases of potential reference invariance and ontological discontinuity. Most commentators have taken as their starting point Laudan's infamous list which, Laudan alleged, could be "extended ad nauseam". It seems that most of the ensuing literature has focused on tackling the cases explicitly mentioned by Laudan, and very few case-studies have been sought beyond the list.

Prima facie, the immense breadth of scientific enterprise over the past couple hundred years (say) bears promise of many more cases to be discussed in order to get a faithful overview of theory change in science. It certainly isn't justified to zoom in on fundamental physics, and ignore the plurality of less fundamental theories, because physics is responsible for only a fraction of our beliefs about the unobservable world. Contemporary philosophy science has rightly started to pay more attention to life sciences and chemistry, for example, in connection with issues such as explanation, scientific method, and evidence, and there is some explicit discussion of scientific realism as well. (Stanford 2006; Turner 2007; Weber 2005) This is the right direction, but much more needs to be done to fully assess the historical evidence for or against scientific realism.

Acknowledging that a much more sweeping scrutiny of the historical record is required immediately raises important questions. For example, it 
isn't immediately clear what kinds of cases one should be looking for as potentially problematic for the realist. Here the debate has been bogged down by the emphasis on ontological discontinuities and referential matters by Kuhn, Laudan, and others. It has not yet been clearly recognised that not all problematic cases need involve ontological discontinuities or non-referring key terms. It is troubling enough for the realist to find past theories that make very accurate novel predictions from false assumptions in such a way that those assumptions are "doing the work" in producing those predictions. These falsehoods need not concern a theory's basic ontology, because realists' commitments with respect to a predictively successful theory (typically) go beyond what the theory says about the furniture of the world. The following example serves to illustrate the issue at stake.

\subsection{Kirchhoff on diffraction}

A relevant case-study - documented in detail in Saatsi and Vickers (2010) concerns Kirchhoff's (1882) prediction of diffraction patterns from radically false and inconsistent premises. I will review just the gist of the case here in order to make the point that ontology and reference can be a red herring regarding the realism debate.

The relevant history of science, in a nutshell, goes as follows. Gustav Kirchhoff derived a celebrated equation in the scalar diffraction theory of optics, describing the behaviour of light with remarkable accuracy. Kirchhoff, like Fresnel, was operating in the ether paradigm, but this in itself is not the cause of trouble for the realist. What's problematic, rather, is that from our current perspective we find that Kirchhoff's derivation hangs on some critical, radically false assumptions regarding the amplitude of light waves. The realist cannot explain Kirchhoff's success by pointing out, for example, that 'ether' in Kirchhoff's theory referred to an idle metaphysical posit that didn't play a role in Kirchhoff's derivation. Although true, this doesn't help the realist here since the challenge is different: the problem is the waves that Kirchhoff had in mind are too far off the target as regards to their amplitudes.

Kirchhoff's derivation turns on some very natural assumptions ${ }^{1}$ that he made regarding the amplitude of light along the closed line integral pic-

\footnotetext{
${ }^{1}$ These are intuitively speaking the simplest assumptions that can be made in the theoretical context in question.
} 


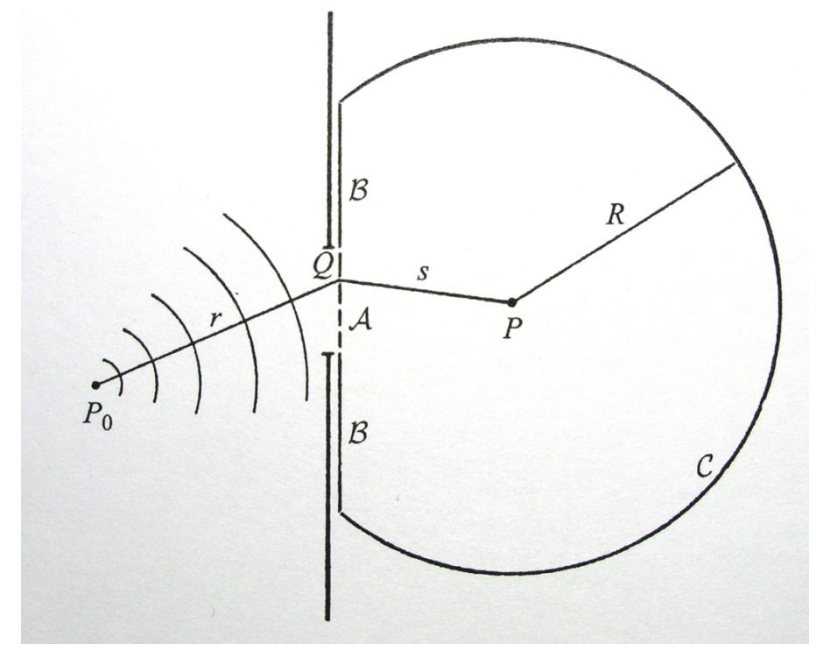

Figure 1: Kirchhoff's line integral.

tured in Figure 1. In particular, he assumed that at the aperture ' $A$ ' the amplitude is as if the screen did not exist, and behind the screen, ' $\mathrm{B}$ ', the amplitude and its normal derivative vanish. The crux of the matter is that Maxwell's theory tells us that in these areas the amplitude of the electromagnetic field is quite different from what Kirchhoff assumed, and we now understand how his erroneous assumptions led him very, very close to the right result. Our contemporary grasp on the physics and mathematics involved gives us understanding of how certain radically different assumptions about wave amplitudes can lead to practically the same end result. There is a kind of 'local underdetermination' realised in Kirchhoff's theorising about diffraction.

Examining the theory more closely yields an understanding of its success, but the case is fundamentally different from many other predictive successes borne out of the research programme of ether optics: in Kirchhoff's case the selective realist is led intolerably astray if she optimistically commits to those premises of Kirchhoff's derivation that are responsible for its success. It is impossible to explain Kirchhoff's success in terms of his key assumptions latching onto reality, viz. his theory being 'approximately true' in some sense, even if the derivation is construed in contemporary terms that ignore the background metaphysical assumption that the ether is the bearer of light waves. 
As far as scientific realism is concerned, cases such as Kirchhoff's teach us that sometimes the realist is led astray if she commits to those assumptions that are responsible for an impressively successful prediction. Most importantly, historical cases in which this can happen need not involve nonreferring terms or radical ontological discontinuity. It is enough that the 'success-fuelling' assumptions are radically wrong about such matters as, for example, what amplitudes of light waves are responsible for producing such-and-such a diffraction pattern.

How common is this type of 'error-tolerance' in science, where some set of natural, simple, yet radically false assumptions lead to essentially the same outcome as a much more complicated theory that is worthy of realist commitment? I don't think we can comfortably answer this question, and this is a shortcoming of the current state of debate. To find examples of successful theories which are not even approximately true one does not need to look for cases of ontological and referential change. This increases dramatically the scope for finding potentially problematic cases. ${ }^{2}$

There are a number of responses that the realist can make to the Kirchhoff case, and it certainly shouldn't be viewed as supporting any kind of wholesale anti-realism. Nevertheless, historical cases of this kind are evidentially probative in evaluating the viability of different realist positions. The case, in and of itself, doesn't speak strongly against the idea that through science we get to know the central features of the world, such as light having a wave-like nature, for example. But scientific knowledge-claims go well beyond such central features in their specificity, and assert things such as "according to the theory the amplitude of light is such-and-such thereabouts". The realist who takes such claims seriously on the grounds of highly successful predictions can be challenged by cases such as Kirchhoff's, which unequivocally demonstrates that occasionally, at least, the simplest, most natural assumptions have led scientists to very impressive predictions despite being radically false.

\footnotetext{
${ }^{2}$ Some successful predictions in old quantum theory may furnish other useful examples of this ilk.
} 


\section{Disparity of explanatory considerations.}

The basic motivation for scientific realism (about theories) is the Miracles argument. The second shortcoming of the current debate has to do with the fact that some key aspects of contemporary realist positions are out of sync with this basic motivation. There are two quite different ways in which this shortcoming is exemplified.

1. Most realist strategies designed to deal with historical discontinuities do not adequately show that the sorts of continuities found underlying historical discontinuities can actually furnish a realist explanation of past predictive successes.

2. A different problem, concerning shifting explanations and explanatory virtues, emerges with the 'explanationist' defence of wholesale realism. This problem harks back to the unresolved challenge of methodological incommensurability which is in tension with the realist's appeal to explanatory virtues as justificatory virtues.

\subsection{Explaining past predictive successes}

Many recent realist positions can be criticised for not being able to explain the successes of past theories any better than a competing anti-realist explanation does. The fact that many realists do not pay due attention to the way in which their position "explains" the predictive successes of past theories is in grave variance with the basic motivation of realism: the idea that that such successes would be "miraculous" if not explained in realist terms.

In order to make this point I will revisit some realist responses to the radical theory-shift from Fresnel's ether theory to Maxwell electromagnetism. The lesson to be learned is that finding continuity between subsequent theories isn't enough for realism: the kind of content found to be continuous across a theory-shift should also be suitably explanatory.

Exhibit A. Worrall's structural realism. Worrall's structural realism, following Poincare, finds evidence for structural continuity in the shift from Fresnel to Maxwell. 
There was an important element of continuity in the shift from Fresnel to Maxwell ... There was continuity or accumulation ... of form or structure, not of content.

This ... seems to offer the only hopeful way of both underwriting the 'no miracles' argument and accepting an accurate account of ... theory change in science.

It is no miracle that [Fresnel's] theory enjoyed the predictive success it did ... because ... it attributed to light the right structure.

The [electromagnetic] field in no clear sense approximates the ether, but disturbances in it do obey formally similar laws to those obeyed by elastic disturbances in a mechanical medium.

Thus, if we restrict ourselves to the level of mathematical equations ... there is complete continuity...

Fresnel's theory [made correct predictions] because it had accurately identified certain relations between optical phenomena.

The [correspondence] principle applies purely at the mathematical level, and is compatible with theoretical assumptions being entirely [different].

Worrall (1989)

There has been much discussion of structural realism's vices and virtues. Here I want to focus on the following aspect that has received less notice. After appropriately appreciating the fact that a realist position should 'underwrite' the Miracles argument, Worrall tries to account for Fresnel's predictive success by saying that it is explained by the fact that Fresnel attributed to light the right structure. This continuity in structure, in turn, is explicated in terms of 'formally similar laws' and 'mathematical equations', expressing 'relations between optical phenomena'. The case in point is the complete continuity from Fresnel to Maxwell in the so-called Fresnel's equations, expressing relative intensities of reflected and refracted polarized 
light.

Has Worrall done justice to the realist's main motivation, the Miracles argument? I think not. Continuity in Fresnel's equations - expressing 'relations between optical phenomena'- provides little more than an illusion of explanation of Fresnel's success, comparable with Molière's virtus dormitiva. Fresnel's predictive success is explained by the fact that he manages to derive the right equation from his theoretical assumptions concerning the nature of light. But the real miracle - how Fresnel managed to derive the right equation from his theoretical assumptions - is thereby left wholly unexplained! To provide an explanation that satisfies the realist intuition one needs to look at the actual derivation that Fresnel employed and the role that Fresnel's theoretical assumptions played in that derivation, and to find suitable continuity between that derivation and Maxwell's theory. It turns out that a requisite level of continuity exists, but talk of formal/mathematical structures doesn't begin to capture it. (Cf. Saatsi (2005) for an account.)

Exhibit B. Chakravartty's semirealism. Anjan Chakravartty, following Worrall, also focuses purely on continuity in Fresnel's equations, and ignores all the theoretical assumptions that Fresnel needed to derive these equations. Chakravartty's (2007) semirealism differs from Worrall in the way he interprets Fresnel's equations as 'getting the structure right'. Chakravartty reads Fresnel's equations as describing relations between certain dispositionsthe so-called 'detection properties' - that are required to give a 'minimal interpretation' of these equations. More generally, it is recommended that

The realist should expect to retain only those structures required to give a minimal interpretation of the mathematical equations used to describe well-established practices of detection, intervention, manipulation, and so on.

Chakravartty (2007)

Fresnel's equations fit the bill, since they describe how one can manipulate light beams of different intensities by reflection and refraction. Chakravartty, too, appreciates the realist's need to underwrite the Miracles argument. He tries to accounts for Fresnel's success by the fact that Fresnel managed to latch onto the relations between some crucial detection properties ('a concrete structure'). 
Given a knowledge of concrete structures it is no miracle ... that good scientific theories are empirically successful, for they describe the structures of reality. (Ibid.)

Again, the worry is that semirealism fails to provide a bona fide explanation of Fresnel's success in a way that satisfies realist intuitions. Admittedly Chakravartty's metaphysics does furnish an explanation of sorts for why Fresnel's theory made right predictions; it made right predictions because the equations that Fresnel managed to derive encode light's disposition to behave in a certain way under certain circumstances, entailing the predictions. Fresnel's theory made right predictions simply by virtue of latching onto these dispositions. This is an explanation, but a superficial one at that, and goes little beyond an alternative empiricist explanation that proceeds in terms of Fresnel's theory being empirically adequate. ${ }^{3}$ The realist's Miracles argument sets forth a deeper explanatory request, however, for the real "miracle" surely is how Fresnel's prima facie false theoretical assumptions about the nature of light allowed him to latch onto those dispositions (or empirical adequacy, for that matter). This is left wholly unaccounted for by Chakravartty's story. To explain away that miracle, it isn't enough to focus on Fresnel's equations; one needs to look at Fresnel's theoretical assumptions and the way in which Fresnel arrived at those equations.

Exhibit C. Ramsey-sentence realism à la Cruse \& Papineau. Pierre Cruse and David Papineau (2002) have advocated a version of Ramseysentence realism according to which 'the empirical success of scientific theories can adequately be explained by appeal to the approximate truth of their Ramsey-sentences.' They argue that the referential success or otherwise of the key terms in a theory is irrelevant to whether or not the theory's success can be explained in realist terms. This is correct, I think. But the way in which they claim to be able to explain a past theory's success seems wrong.

Faced with an empirically successful theory, the realist can argue, not to the approximate truth of the theory itself, but to the approximate truth of its Ramsey-sentence. (p. XX, my emphasis)

\footnotetext{
${ }^{3}$ Chakravartty's explanation in terms of dispositions is metaphysically loaded, of course. But this in itself does not make it a realist explanation that lives up to the intuition behind the Miracles argument.
} 
The idea is that the approximate truth of a theory's Ramsey-sentence explains its success, and the critical notion of 'the approximate truth of a Ramsey-sentence' is then (somewhat vaguely) explicated as the requirement that existential quantifiers of a Ramsey-sentence pick out something in the world which has most of the properties and relations attributed to it by the Ramsey-sentence. For example:

Suppose "the aether" fails to refer to anything. Then there is no question of the aether possessing any properties, approximately or otherwise. But this does not preclude the assessment of the relevant existential Ramsey-sentence for approximate truth. It could be approximately true that there is an entity which is the seat of electromagnetic phenomena, and involves transverse radiation, and ... consists of an elastic solid. After all, there is indeed something which is the seat of electromagnetic phenomena, and involves transverse radiation, and so on-namely, the electromagnetic field - even if it is not an elastic solid.

This proposal is problematic from the word 'go'. The explication of the approximate truth of a Ramsey-sentence does nothing to ensure that the properties and relations that get carried over in theory change are actually explanatory of the predecessor theory's success. That is, it is possible that (i) two theories agree on most things they say about some unobservable entity-type, but that (ii) it turns out that the disagreements are critical for explaining the predecessor theory's predictive success, whilst the agreements are more or less immaterial for that explanation. ${ }^{4}$

To sum up, the lesson to draw from the above review of recent realist positions is this. It isn't enough for the realist to find a way to capture continuity across theory-shifts. One also needs to keep the basic realist motivation firmly in mind and make sure that any continuity found in theory change underwrites a bona fide explanation of the relevant past successes.

\footnotetext{
${ }^{4}$ For example, the fact that Fresnel construed light as wave motion of the ether turns out to be somewhat immaterial for understanding how Fresnel managed to derive his equations. (Saatsi, 2005)
} 


\subsection{Shifting explanations and explanatory virtues}

Even if we can find continuity in radical theory-shifts that furnishes a realist explanation of past predictive successes, these theory-shifts may still pose a problem for the Miracles argument. The reason is that in many instances of theory change there is radical discontinuity in the explanations that the respective theories give of their target phenomena. The Miracles argument is often portrayed as being on a par with various scientific instances of inference to the best explanation. Anti-realists who are sceptical of scientific IBEs criticise the argument as question begging (e.g. Laudan, 1981). In response, some realists have defended the Miracles argument as being "merely" rulecircular, as opposed to premise-circular (Psillos, 1999). Regardless of the outcome of that debate, the realist must acknowledge that there is still a significant, undeniable sense in which inference to the best explanation has led to the wrong conclusion many times in the history of science. The existence of the ether that waves thus-and-so was the best explanation of diffraction phenomena. The existence of the caloric with such-and-such properties was the best explanation of the speed of sound. And so on. The 'selective' realist's strategy - the famous 'divide et impera' move - of focusing selectively on those elements of past theories that both (i) get carried over in theory change, and (ii) are explanatory of past predictive successes from our current perspective, completely fails to address this issue of discontinuity with respect to scientific explanations. (cf. Doppelt, 2005) If the Miracles argument is defended as being on a par with scientific IBEs, then it seems that the best the realist can say is that the conclusion of the Miracles argument- the explanans of this particular instance of inference to the best explanation - is latching onto reality to the extent that is required to explain its predictive success. And the obvious worry now is that the realist hypothesis makes no empirical predictions different from those made by an alternative anti-realist hypothesis that says, for example, that scientific IBEs and predictive success are a sign of empirical adequacy. ${ }^{5}$

\footnotetext{
${ }^{5}$ This tension between the explanationist defence of realism, on the one hand, and the selective realist response to the history of science, on the other, has been noted by Doppelt 2005. His recent novel twist on the explanationist defence of realism is premised on the assumption that part of the explanandum of the Miracles argument is the explanatory success of science. (Doppelt 2007) From my perspective past explanations in terms of the ether or the caloric, for example, were not successful, since they were false. More would have to be said on this, of course, to fully respond to Doppelt's subtle argument.
} 
There is a further dimension to this challenge to the Miracles argument, due to the fact that arguably there are also shifts in the explanatory virtues (in addition to shifts in particular scientific explanations). (E.g. de Regt and Dieks, 2005) Acknowledgment of such "meta-level" discontinuities harks back to Kuhn's discussions of paradigm shifts and methodological incommensurability, of course. Over the last couple of decades the scientific realism debate has been mainly driven by Laudan's challenge to realismformulated in terms of reference variance - according to which the history of science allegedly shows that predictive success is not correlated with theories' approximate truth. The selective realist's response to that challenge has been quite convincing on the whole, I think, but a broadly Kuhnian challenge remains. If the history of science really displays transitions from one explanatory paradigm to another - shifts on how explanations are evaluated and compared ${ }^{6}$ - then the meta-inductive support for the Miracles argument is weakened yet further. For arguably the best scientific thinkers of one paradigm often fail to appreciate the explanations that are deemed to be the best explanations by scientists operating within a different methodological paradigm. In light of this, the realist's preference for her explanation of the predictive success of science is naturally understood in terms of the realist occupying a different "paradigm" from her opponent. The realist may feel that she is able to objectively compare different explanations for the success of science, but why would a philosopher of science be in a different epistemic boat from the scientist, as a cognitive agent evaluating IBEs? After all, the realist's (rule-circular) defence of the Miracles argument underlines the fact that in relation to the IBE-reliant method of science the Miracles argument is just "more of the same".

It may well be possible to construct a good argument on the basis of the Miracles intuition, but one lesson from the history of science is that this cannot be achieved simply by arguing that IBE is a reliable mode of inference as testified by its role in successful scientific reasoning.

\section{Over-generalisation}

Philosophers like to make sweeping generalisations and often hope to find far-reaching and exhaustive uniformity in the world. Regularly it turns out,

\footnotetext{
${ }^{6}$ Examples here....
} 
however, that such hopes are in vain as allegedly uniform subject matters break down into a plurality of cases each one of which requires individual attention to detail. Like so many other problems in philosophy, the question of realism has been posed in extremely general terms at the outset, and considerations such as the Miracles argument have been put forward in the hope to secure realism about "all or most of mature science" in one fell swoop. I will now propose that the realism debate can progress by renouncing such extreme level of generality in its arguments and acknowledging the heterogeneity and the multifaceted nature of theoretical science, and by studying our epistemic commitments in a more piecemeal way.

David Papineau suggests a piecemeal approach to the realism debate in his introduction to (Papineau, ed., 1996).

One issue ... is whether different philosophical morals may apply in different areas of science. Perhaps we should be fundamentalists in physics but not biology. Or perhaps we should be theory realists in chemistry, entity realists in geology, and outright sceptics in paleobiology. ... Perhaps a more fine-grained approach would be worth the extra effort. Now that we are clear about the epistemological options on offer, there is no obvious reason why we should expect the same alternative to apply to every scientific discipline.

(Papineau, 1996)

Papineau's suggestion makes perfect sense but for some reason it hasn't been widely heralded in the literature; as far as I can see, neither Papineau nor anyone else has been willing to put in 'the extra effort'. Rather, different forms of realism and anti-realism are typically presented as epistemic positions that are applicable across the board. But one can go even further than Papineau does in the above quote, and argue that there is reason not to expect the same alternative to apply to every scientific discipline. I'll finish the paper with some remarks to this end vis-a-vis the issue of historical evidence.

It is critical to acknowledge that scientific enterprise on the whole exhibits variation in many respects relevant for the realism debate. There are substantial features of explanatory practice and evidential considerations 
in science that differ from one scientific domain to another. For example, explanation in molecular biology and neuroscience, say, is arguably a matter of producing suitable causal-mechanical accounts, whilst in fundamental physics much of explanation is non-causal. ${ }^{7}$ When it comes to linking evidential considerations to explanatory value (IBE), it may well be that more abstract non-causal explanations of quantum physics, say, are much less reliable in tracking the truth compared to causal-mechanistic explanations. Given a particular domain of scientific theorising, with particular desiderata for good explanation, we may look for historical evidence for the unreliability (or otherwise) of IBE within that domain. Perhaps an extensive overview of the historical record will show that practically all cases that speak against the Miracles intuition pertain to specific domains of science, indicating that a degree of pessimism is well justified with respect to our current theoretical beliefs in these domains. Perhaps we will come to understand-by reflecting on the methods and subject matters of these domains - how prima facie impressive predictive success (eliciting the Miracles intuition) is actually quite achievable from radically false premises due to a kind of local underdetermination. Kirchhoff's prediction is a case in point here. (Saatsi and Vickers, 2010)

The problem of over-generalisation stems from careless extrapolation from a very limited number of historical case-studies. It should be easy to appreciate the basic intuition that a case-study that concerns a model of cosmology that has made impressive novel predictions from apparently false assumptions - should such a case be discovered - might not speak at all against realism about molecular genetics, given the obvious differences in the respective domains. We can battle over-generalisation in the realism debate by forming and arguing for more tightly delineated philosophical positions. In practice this requires that the basic arguments for and against realism - including the arguments turning on historical evidence - get relativized to particular domains of science with their particular subject matters, problems, and methods. Instead of defending "(anti-)realism about all (or most) mature science" one should focus one's ambitions to "(anti-)realism of kind $K$ about theoretical beliefs supported by evidence of type $E$ in a domain $D$ ", where the variables allow for variability in features that support

\footnotetext{
${ }^{7}$ Cf. Bechtel and Abrahamsen (2005) and Craver (2007) versus Bokulich (2008), for example.
} 
more nuanced treatment of historical and philosophical evidence.

\section{References}

Bechtel, W. and Abrahamsen, A. (2005). Explanation: The Mechanist Alternative. Studies in History and Philosophy of the Biological and Biomedical Sciences, 36:421-441.

Bokulich, A. (2008). Can Classical Structures Explain Quantum Phenomena? British Journal for the Philosophy of Science, 59(2):217-235.

Chakravartty, A. (2007). A Metaphysics for Scientific Realism. Cambridge University Press, Cambridge.

Craver, C. F. (2007). Explaining the Brain. Oxford University Press, Oxford.

Cruse, P. and Papineau, D. (2002). Scientific Realism Without Reference. In Marsonet, M., editor, The Problem of Realism, pages 174-189. Ashgate, Aldershot.

De Regt, H. W. and Dieks, D. (2005). A Contextual Approach to Scientific Understanding. Synthese, 144:137-170.

Doppelt, G. (2005). Empirical success or explanatory success: What does current scientific realism need to explain? Philosophy of Science, 72(5):1076-1087.

Doppelt, G. (2007). Reconstructing scientific realism to rebut the pessimistic meta-induction. Philosophy of Science, 74(1):96-118.

Laudan, L. (1981). A Confutation Of Convergent Realism. Philosophy of Science, 48:19-49.

Magnus, P. and Callender, C. (2004). Realist ennui and the base rate fallacy. Philosophy of Science, 71(3):320-338.

Papineau, D. (1996). Introduction. In Papineau, D., editor, The Philosophy of Science, chapter 1, pages 1-21. Oxford University Press.

Psillos, S. (1999). Scientific Realism: How Science Tracks Truth. Routledge, London. Ch.4.

Saatsi, J. and Vickers, P. (2010). Miraculous success? Inconsistency and untruth in Kirchhoff's diffraction theory. The British Journal for the Philosophy of Science. Forthcoming. 
Saatsi, J. T. (2005). Reconsidering the Fresnel-Maxwell case study. Studies in History and Philosophy of Science, 36:509-538.

Stanford, K. P. (2006). Exceeding Our Grasp. Oxford University Press, Oxford.

Turner, D. (2007). Making Prehistory: Historical Science and the Scientific Realism Debate. Cambridge University Press.

Weber, M. (2005). Philosophy of Experimental Biology. Cambridge University Press, Cambridge.

Worrall, J. (1989). Structural Realism: The Best Of Both Worlds? Dialectica, 43:99-124. Reprinted in Papineau, D. (ed.) The Philosophy of Science (Oxford: Oxford University Press), pp. 139 âĂŞ 165. 